A. Is ebree Moens: Der erste Wellengipfel i. d. absteigenden Schenkel etc. $\mathbf{5 1 7}$

(Aus dem physiologischen Institut der Universität Leiden.)

\title{
Der erste Wellengipfel in dem absteigenden Schenkel der Pulscurve.
}

$$
\text { Von }
$$

\section{Dr. A. Isebree Moens \\ in Goes (Holland).}

Mit 3 Holzschnitten.

In meiner im Jahre 1878 publicirten Arbeit „Ueber die Puls-

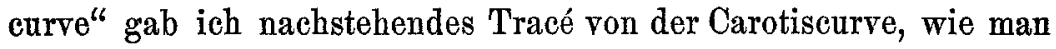
dieses beim gesunden Menschen unter günstigen Verhältnissen erhalten kann.

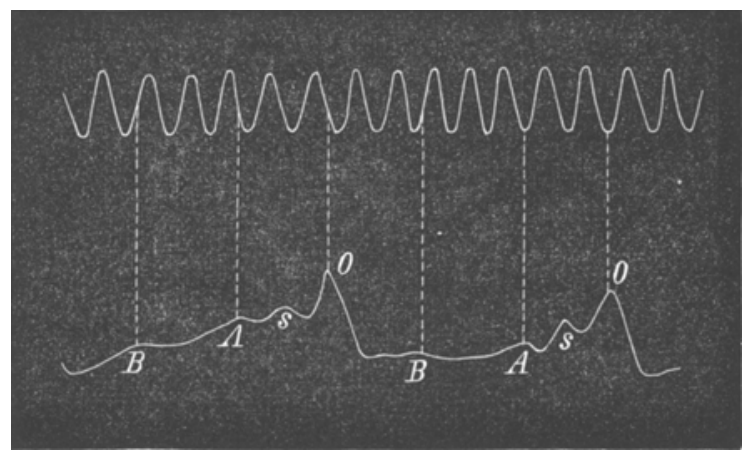

Hig. 1.

Ausser der primären Elevation $o$, findet man regelnässig in dem absteigenden Schenkel drei Gipfel. Zwei dieser Gipfel $A$ und $B$, lernte ich als ersten und zweiten Schliessungsgipfel kennen. Ich bewies dabei, dass diese Gipfel nicht durch ein eigenthümliches Zusammenziehen des Herzens verursacht werden, sondern dass sie auch in einer elastischen Röhre entstehen, deren eines Ende vermittelst eines Hahnes mit einem Druckgefäss verbunden und deren anderes Ende in ein Reservoir getaucht ist. Wenn nämlich die Flüssigkeit durch die Röhre strömt und der Hahn 
plötzlich geschlossen wird, so entsteht am geschlossenen Ende der Röhre und in gleichmässigen Zwischenräumen eine Reihe Wellen, die sich mit einer den Wellen eignen Geschwindigkeit in der Röhre fortpflanzen. - Die von mir gegebene Erklärung ihres Entstehens ist kurzweg folgende: beim Schliessen des Hahnes fällt die ausgedehnte Röhre successive über ihre ganze Länge von dem geschlossenen Ende an zusammen; der Druck, den die Wand der Röhre, ehe derHahn geschlossen wird, auf den Inhalt ausübt, wird nun durch eine saugende Kraft ersetzt, deren Werth je nach dem Punkte der Röhre verschieden ist. Die Resultirende dieser Saugkräfte'bringt die noch stets weiterströmende Flüssigkeit allmählig zum Stehen, um sie darauf wieder in die Röhre zurückzuziehen. Die Röhre füllt sich allgemach, und hierdurch verringert nach und nach die Saugkraft der Wand, bis dieselbe wieder positiven Druck auszuïben beginnt. Bald darauf genügt dieser Druck zum Heraustreiben des Inhaltes aus der Röhre. Bei diesem Wendepunkt in der Bewegung der Flüssigkeit entsteht der erste Schliessungsgipfel.

Ich berechnetc die Spannkräfte, die sich beim Füllen und beim Zusammenfallen der Röhre in deren Wand entwickeln und leitete davon die Zeitdauer ab, die zwischen dem Schliessen des Hahnes und dem Entstehen des ersten Schliessungsgipfels vergehen muss; der erlangte Werth ist S. 48 angegeben; diese Zeitdauer ist eine Function des specifischen Gewichtes der angewandten Flüssigkeit, des Durchmessers und der Länge der Röhre, der Dicke and des Elasticitätscoefficienten ihrer Wand. Das vollkommene Uebereinstimmen der Zahlen, die bei verschiedenen elastischen Röhren durch Anwendung der gefundenen Formel und auf experimentellem Wege erlangt wurden, beweist die Richtigkeit meiner Auffassung.

Mein Studium erstreckte sich iiber ein verzweigtes, elastisches Rohrsystem, und ich gelangte dabei zu analogen Resultaten: auch hier entsteht beim Schliessen des Hahns eine Wellenreihe, die über das Röhrensystem hinläuft. Diese Wellen nannte ich Schliessungswellen.

Wie in dem verzweigten, elastischen Rohrsystem eine Reihe Schliessungswellen vorkommen, so muss dies auch der Fall sein in dem arteriellen Gefässsystem durch das Aufhören der Blutzufuhr (also sobald das Einfliessen in die Aorta aufhört, was dem Schliessen des Hahnes in meinen Experimenten mit elastischen 
Der erste Wellengipfel in dem absteigenden Schenkel der Pulscurve. 519

Röhren entspricht); ich bewies: dass die Gipfel $A$ and $B$ in dem absteigenden Schenkel des Pulses die erste und die zweite Schliessungswelle des arteriellen Gefässsystems sind.

Ich widerlegte in meiner Arbeit Marey's, Ludwig's, Onimus und Viry's und Rive's Ansichten, die den dicrotischen Gipfel durch die Reflexion der primären Erhebung zu erklären suchten.

Lando is' Verdienst erkannte ich, in so weit er die Identität der Gipfel $A$ und $B$ mit den Schliessungswellen (seine Riickstosselevationen) dargethan hat. Dahingegen bestritt ich die von ihm aufgestellte Erklärung dieser Wellen. Lando is behauptet nämlich: dass in einer elastischen Röhre, nachdem der Hahn geschlossen, der Inhalt durch Contraction der Rohrwand, also durch den Druck dieser Wand auf die Flüssigkeit, nach dem geschlossenen Ende getrieben werde, während ich deutlich bewies, dass es nicht der Druck sondern das Saugen der Rohrwand ist, das der Flissigkeit diese Bewegung gibt.

Am schwersten liess sich der Gipfel $s$ erklären. Ich schloss, dass dieser ein partieller Schliessungsgipfel (Ma r ey's „onde secondaire") sei. Obgleich es mir nicht gelang allen Eigenschaften dieser Wellen nachzuforschen, so konnte ich doch Folgendes mit Gewissheit bestimmen:

1. Die Erhebung wird sowohl in einer einzelnen unverzweigten elastischen Röhre als in einem verzweigten Röhrensystem nach dem Schluss des Hahnes wahrgenommen.

2. Sie entsteht wie die Schliessungswellen am geschlossenen Ende der Röhre und pflanzt sich von hier mit einer den Wellen eignen Geschwindigkeit peripherisch fort.

Es ist besonders der erste Satz, der mir sehr wichtig erscheint, weil dadurch erwiesen wird, dass die Erklärung des Gipfels $s$ nicht in einer absatzweise stattfindenden Contraction der Herzkammer zu suchen ist. In meinen Experimenten mit elastischen Röhren $\left.{ }^{1}\right)$ kam ja die Erhebung $s\left(s^{*}\right.$ und $s^{\prime \prime}$ Fig. 2) zu Stande, nachdem der Hahn geschlossen war $\left(o, o^{\prime}\right.$ und $o^{\prime \prime}$

1) Vgl. meine Abhandlung über die Pulscurve, S. 31. 
Fig. 2): es kann also hier nicht die Rede sein von einer in zwei Tempo's stattfindenden Schliessung.

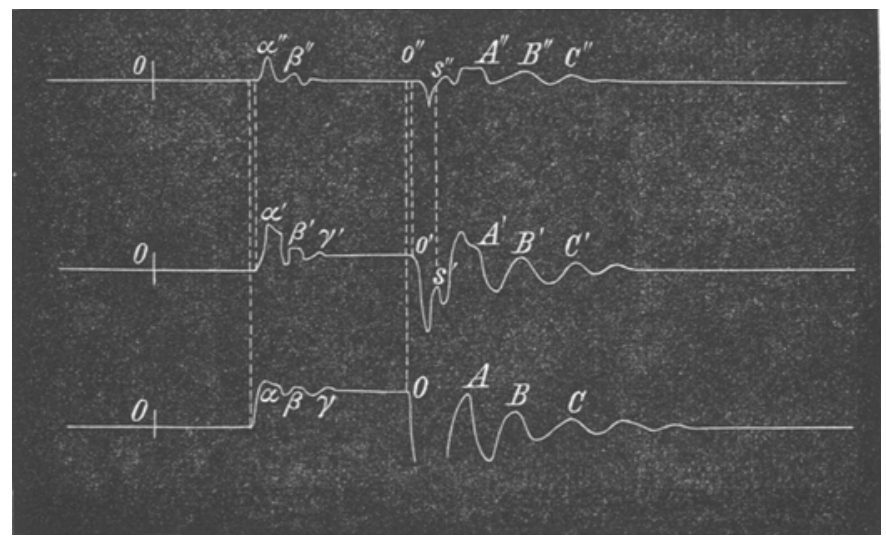

Fig. 2.

Es wurde von Traube (Ges. Abh., Bd. III, S. 595) eine solche absatzweise stattfindende Contraction der Herzkammer zur Erklärung der zwei ersten Elevationen in dem absteigenden Schenkel (meine Gipfel $s$ und $A$ ) wirklich angenommen, allein er gibt keinen einzigen genügenden Grund für diese Hypothese an.

Rosenstein (Deutsch. Arch.f. kl. Medicin, Bd. XXIII) stimmt zum Theil mit dieser Theorie überein. Er gesteht zwar, dass Traube sich irrt, wo er die beiden Gipfel und also auch den dicrotischen Gipfel $A$ von der absatzweise stattfindenden Herzcontraction ableitet. Rosenstein glaubt jedoch Traube hinsichtlich der ersten secundären Erhebung Recht geben zu müssen (Gipfel s), und zur Erklärung dieser Meinung nimmt er ebenfalls an, dass wirklich die Contraction der Herzkammer absatzweise zu Stande kommt.

Rosenstein sucht die doppelte Contraction der Herzkammer zu beweisen durch die doppelte Erhebung, welche er beim Registriren des Herzschlages bemerkt, sogar wenn die arteriellen Gefässstämme des Herzens unterbunden sind. Dass wirklich die erste secundäre. Erhebung $(s)$ in der Carotiscurve durch den zweiten Gipfel der doppelten Elevation in dem Cardiogramm verursacht wird, hält Rosenstein für bestätigt durch das Fehlen des Gipfels $s$ in der Carotiscurve bei abortiven Herzschlägen, wobei die Herzcontraction nicht absatzweise stattfinden sollte.

Ich beabsichtige nicht auf eine Beurtheilung von $R$ os enstein's 
Cardiogrammen einzugehen, glaube mich aber zu dem Schluss berechtigt, dass wenn auch beim Registriren des Herzschlages eine doppelte primäre Erhebung vorgefunden wird, doch noch nicht dadurch der Beweis geliefert ist, dass die Contraction der Herzkammer absatzweise geschieht. - Und wenn auch solch eine doppelte Contraction der Herzkammer wirklich bewiesen wäre, so könnte sie doch noch unmöglich zur Erklärung der ersten secundären Elevation dienen. In der von Rosenstein S: 96 gegebenen Curve, in welcher die Zeit registrirt wird, findet ja die erste secundäre Erhebung bei der Carotiscurve des gesunden Menschen, 0,2 Sek. statt, nachdem das Blut angefangen hat in die Aorta zu fliessen (wie in meinem Tracé, wo $s$ ebenfalls 0,2 Sek. von $C$ entfernt ist; vgl. Fig. 3). Nachher werde ich beweisen, dass das Einströmen des Blutes in die Aorta unmöglich länger als 0,1 Sek. dauern kann, und also das Herz schon entleert ist, bevor der erste secundäre Gipfel entsteht, weshalb eine zweite Contraction des Herzens, wenn diese auch Statt fände, unmöglich diesen Gipfel verursachen $\mathrm{k} \ddot{\mathrm{n} n \mathrm{n}} \mathrm{te}$.

Hinsichtlich des Verschwindens der Erhebung $s$ bei abortiven Herzschlägen wïnsche ich zu erwähnen: dass diese in der von Rosenstein von der Carotis erhaltenen Curve (S. 96) keineswegs fehlt, sobald die Herzschläge abortiv waren, sondern nur weniger deatlich ist. Später will ich beweisen, dass dies ganz mit meiner Erklärung des Gipfels $s$ übereinstimmt. Dieser Gipfel muss bei schwachen Herzschlägen schwächer werden, und Rosenstein ist also im Irrthum, wenn er im Fehlen dieses Gipfels eine Bestätigung seiner Theorie sieht.

Marey (Circulation du sang, 1863, p. 273 et 274) bemerkt: dass nach der primären Erhebung Blut nach dem Herzen zurïckfliesse, wodurch die Valv. semil. geschlossen werden. Dieses Schliessen sollte den Gipfel $s$ erzengen.

Auch Landois meint, der Gipfel sei aus dem Schliessen der Semilunarklappen entstanden. Dadurch entstehe dort, wie er behauptet, eine Welle; allein er gibt nicht an, wodurch sich die Klappen schliessen. - Schon früher war ich der Meinung: dass die Semilunarklappen sich zwar gleichzeitig mit der Bildung des Gipfels schliessen, dass dies aber nicht für die Ursache dieses Gipfels, sondern für eine Folge seiner Entstehung zu halten 
ist. Landois gibt also von der Bildung dieses Gipfels keine Erklärung.

Ich will versuchen im Nachfolgenden den Ursprung des Gipfels $s$ näher zu erklären; werde jedoch eine kurze Betrachtung uiber die Systole des Ventrikels vorangehen lassen, und zugleich die Folgen untersuchen, welehe die Blutbewegung aufs Herz selbst ausïben muss, weil diese mit der Bildung des Gipfels eng zusammenhangen.

Systole jeder Herzabtheilung heisst man jenen Zustand des Herzens, in welchem deren Muskelfasern thätig sind; Diastole heisst jener Zustand, in welchem die Muskeln erschlafft sind.

Die Dauer der Ventrikelsystole ist beim erwachsenen Menschen ziemlich constant und nach Donders' Untersuchungen im Durchschnitt 0,314 Sek.; nach L and o is (Arterienpuls, 1872, S. 307) ist die Thätigkeitsdauer der Ventrikelwand $=0,258$ Sek., nämlich die Summe seiner Nummern: 4, 6 und 3 (Dauer vom Beginne der Contraction des Ventrikels bis zur Eröffnung der Semilunarklappen $=0,085$ Sek., Daner der Systole des Ventrikels nach Eröffnung der Semilunarklappen $=0,088$ Sek. und Dauer des Verharrens in der Contraction $=0,085$ Sek.).

Das eigentliche Resultat dieser Kammersystole ist das Einpumpen des Inhaltes in die Aorta. Die Dauer dieser Blutbewegung selbst ist viel kiirzer als die der eigentlichen Systole; denn erstens hat die Systole schon angefangen, ehe das Blut sich in Bewegung setzt und zweitens hat diese Bewegung schon ganz aufgehört bevor die Erschlaffung der Muskelfasern anfängt. Der Grund der unter erstens genannten Zeitdauer ist folgender: die Muskelfasern der Herzkammer ziehen sich nach einander zusammen, auch ist die Kraft, mit weloher jede Muskelfaser sich contrahirt, im Anfange nur gering und erlangt erst nachher ihre volle Thätigkeit, und endlich muss die Ventrikelwand schon hinreichend Arbeit geleistet haben, um den Inhalt unter höhern Druck zu versetzen, als unter welchem das Blut in der Aorta steht ${ }^{1}$ ). Sonst

1) Wirklich beweisen die Versuche von Goltz und Gaule (Pflüger's Arch., Bd. XVII), dass der Maximaldruck im linken Ventrikel grösser ist als der im Beginne der Aorta. 
Der erste Wellengipfel in dem absteigenden Schenkel der Pulscurve. 523

können ja.die Valvulae semilunares nicht geöffnet werden. Dies bedarf einiger Zeit, welche von Landois gemessen ist; er fand dafür $=0,085$ Sek.

Die Ursache der unter zweitens genannten Zeitdauer ist diese: das Einströmen des Blutes in die Aorta und die Systole (wenigstens in Betreff ihrer Dauer) sind von einander ganz unabhängig: die Ursache der Blutbewegung wird ja durch die Intensität der Herzeontraction '), des sämmtlichen in der Ventrikelhöhle enthaltenen Blutes und durch die Spannung in der Aorta bedingt; die Dauer der Systole dahingegen hängt hauptsächlich von der Zuckungszeit der Muskelfasern ab.

Die Zeit, welche der Ventrikel zum Austreiben seines Inhaltes braucht, wird von verschiedenen Forschern angegeben. So sagt Rive-(Dissertatio, S. 71): „dat onmiddellijk bij de opening der Valvulae semilunares de grootste hoeveelheid bloed in de slagader gedreven wordt." Da sie nach Rive während 0,108 Sek. Statt findet, so kann ja die Zeit des Ausströmens fast nicht länger als 0,108 Sek. dauern.

Davon sagt Landois (1. e., S. 3レ6): „dass 0,227 Sekunden hindurch das Einströmen des arteriellen Blutes in die Aorta hinein dauert." Dies ist zugleich nach Land ois der Zeitraum, der zwischen dem Eröffnen und dem Zuschliessen der Semilunarklappen vergeht: in dieser Zahl ist auch der Werth seiner Nummern 3 und 7 begriffen („Dauer des Verharrens in der Contraction" und „Dauer vom Beginne der Erschlaffung der Muskelfasern des Ventrikels bis zum Schluss der Semilunarklappen“, vgl. 1. c., S. 307); in keiner dieser beiden Perioden kann jedoch Blut aus dem Ventrikel in die Aorta gepumpt werden. Wird hierauf Rücksicht genommen, so folgt aus Lando is' eignen Zahlen, dass die Periode der Blutbewegung nur 0,088 Sek. dauert ${ }^{2}$ ).

1) Je stärker z. B. die Herzcontraction, desto grösser caet. par. die Geschwindigkeit, mit welcher der Inhalt in die Aorta gepumpt wird and desto kürzer also die Zeit, in der dies Statt findet.

2) Aus Landois' Lehrbuch d. Physiologie, 1879, S. 95 scheint hervorzugehen, dass auch er jetzt ungefähr 0,088 Sek. für die Dauer des Einströmens annimmt. Schade jedoch ist es, dass durch einen Druckfehler statt jener Zahl hier $0,8-0,9$ Sek. gefunden wird. 
Heynsius („Ueber die Ursachen der Töne und Geräusche", 1878, S. 51) fand dafür 0,1 Sek.

Wenn wir nun im Durchschnitt 0,3 Sek. für die ganze Systole nehmen, 0,085 Sek. für deren ersten Zeitabschnitt (während dessen noch kein Blut in die Aorta getrieben wird), und wenn wir 0,1 Sek. setzen für die Periode der eigentlichen Blutbewegung, so bleibt für die letzte Periode der Systole (während welcher der linke Ventrikel entleert ist und zugleich zusammengezogen bleibt) noch 0,115 Sek. übrig. Lando is fand für diese Periode, seine „Dauer des Verharrens in der Contraction", 0,085 Sek.

Aus Folgendem erhellt die grosse Wichtigkeit dieser letzten Periode der Systole für die Blutbewegung, weil während dieser Periode ein leerer Raum im Ventrikel entsteht, den ich für die wichtigste Ursache des Gipfels $s$ halte.

Im Nachstehenden unterscheiden wir:

1. diesen leeren Raum,

2. dessen Einfluss auf die Blutbewegung,

3. die Anwendung des Vorhergehenden auf die Carotiscurve.

1. Der nach dem Einströmen des Blutes in die Aorta in der Herzkammer entstandene leere Raum.

Schon früher habe ich gesagt, dass man sich von dem Einströmen des Blutes aus der Herzkammer in die Aorta eine Vorstellung: bilden kann, wenn man eine elastische Röhre nimmt, deren eines Ende geöffnet und deren anderes vermittelst eines Hahnes mit einem Druckgefäss verbunden ist. Es kann die Veränderung des Rohrdiameters an verschiedenen Stellen durch Cardiographen angezeigt werden; auf diese Weise erhält man einen Massstab des Druckes, welcher in der Röhre statt findet: Erhöhung des Druckes ist ja mit Vergrösserung des Rohrdiameters verbunden, während bei Abnahme desselben der Diameter kleiner wird oder die Röhre zusammenfällt. Wenn der Hahn geöffnet ist, also die Flüssigkeit aus dem Druckgefäss durch die Röhre strömt und jener plötzlich geschlossen wird, sinken unmittelbar darauf die Hebel aller Cardiographen nach einander; das Sinken ist so bedeutend, dass daraus erhellt, dass nach dem Schliessen ein negativer 
Der erste Wellengipfel in dem absteigenden Schenkel der Pulscurve. 525

Druck in der Röhre entsteht. Die Curven, welche die Hebel dabei auf einen drehenden Cylinder notiren, beweisen uns zugleich, wie kurze Zeit der negative Druck gedauert hat und wie er sich von dem Hahne an "über die ganze Röhre verbreitet. Er wird durch das plötzliche Aufhören des Fliessens verursacht.

Die Systole des Ventrikels wird noch besser vorgestellt, wenn man das Druckgefäss und den Hahn, wovon die Rede war, dureh eine compressible hohle Kngel ersetzt. Ich nahm einen weissen Cautschuc-Ballon, der 2 Oeffnungen hatte; durch die eine dieser Oeffnungen wurde er mit dem Ende einer Röhre verbunden, während das andre Ende der Röhre in ein Reservoir getancht war. Mit der zweiten Oeffnung des Ballons war ein Quecksilbermanometer vermittelst einer sogenannten Minimalklappe verbunden: eine solche Klappe kann sich nur nach der innern Seite, also nach dem Ballon zu öffnen und dies nur, wenn ein negativer Druck im Ballon entsteht; das Quecksilberniveau des Manometers kann also nicht steigen, sondern nur sinken. Weil das Quecksilberniveau durch das Schliessen der Klappe zugleich seinen niedrigsten Stand beibehält, so lernen wir daraus den grössten negativen Druck im Ballon kennen. In Folge der Klappe wird ein positiver Druck im Ballon natïrlich keinen Einfluss auf den Manometerstand ausüben. Der Ballon und die Röhre waren bei den Experimenten mit Wasser gefüllt. Der Ballon suchte, nachdem er zusammengedrïckt worden war, sobald er freigelassen wurde, seine runde Gestalt wieder anzunehmen, und sog demnach Wasser ein: er besass also eine ihm eigene (statische) Saugkraft; in meinen Experimenten war es wichtig (wie sich nachher ergeben wird), einen negativen Druck im Ballon unabhängig von dieser ihm eigenen (statischen) Saugkraft nachzuweisen. Letztere musste also eliminirt werden; ich erreichte dies, indem ich den Ballon in einer Höhe $h$ über dem Niveau des Reservoirs befestigte, so zwar, dass eine Wassersäule von gleicher Höhe der Saugkraft des Ballons das Gleichgewicht hielt, wodurch diese also eliminirt wurde.

Sobald der Ballon stark zusammengedrïckt und also dessen Inhalt in die Röhre gepumpt worden war, senkte sich unmittelbar darauf das Nivean des Manometers: wodurch bewiesen wird, dass in dem Ballon unmittelbar nach dessen Entleerung ein negativer Druck entsteht. 
Wo ist der Grund dieses negativen Druckes zu suchen? Die dem Ballon eigne Saugkraft ist davon die Ursache nicht, denn diese wurde eliminirt. Sie lässt sich nur aus dem plötzlichen Einströmen des Balloninhaltes in die Röhre erklären.

Die Geschwindigkeit, mit welcher die Flüssigkeit in die Röhre getrieben wird, beträgt im Anfang 0, nimmt stets zu und erlangt ihren höchsten Werth in dem Augenblick, da das $\mathrm{Zu}$ sammendrïcken des Ballons endet; der Zufluss des Wassers hört jedoch nun plötzlich auf, weil der Ballon leer ist. Die in die Röhre getriebene Masse bewegt sich aber weiter und hierdurch muss unmittelbar hinter ihr (also im Ballon) plötzlich ein negativer Druck entstehen.

Die Ausströmungsgeschwindigkeit des Balloninhaltes ist grösser und also der negative Druck im Ballon auch bedentender, wenn derselbe stark zusammengedrückt wird; sie ist geringer, wenn es leise oder langsam geschieht.

Mit einem Ballon, dessen Diameter circa $6 \mathrm{~cm}$ betrug, erhielt ich bèi starkem Zusammendrücken einen negativen Druck von ungefähr $8 \mathrm{~cm}$ Quecksilber; wurde er leise zusammengedrïckt, so änderte sich das Quecksilberniveau nicht.

Der im Ballon entstandene negative Druck verursacht, dass die wenige Flüssigkeit, welche noch in seiner Höhle zurückgeblieben ist, ebenfalls in die Röhre getrieben wird; die innern Wandungen des Ballons liegen jetzt aufeinander und werden sogar in Folge des negativen Druckes fest aneinander gepresst; der negative Druck verliert sich jedoch bald durch die hierdurch verbrauchte Arbeit, und die Dauer ist deshalb nur sehr kurz. Er währt jedoch so lange, dass er einen kleinen Theil des Rohrinhaltes zum Stillstand bringen und später in den Ballon zurüickziehen kann.

Genau dasselbe muss in der Herzkammer nach dem Entleeren ihres Inhaltes Statt finden. Der einzige Unterschied zwischen einem Cautschuc-Ballon und dem Ventrikel ist folgender: ersterer wird durch eine mechanische Kraft ausserhalb desselben zusammengedriickt, während beim letztern die Wand selbst die Kraft ausübt: und dies er Unterschied wird ja weder Einfluss auf die Bewegung des Inhaltes noch auf deren Folgen ausüben. Auch 
Der erste Wellengipfel in dem absteigenden Schenkel der Pulseurve. 527

beim Herzen wird der Inhalt des Ventrikels mit wachsender Geschwindigkeit in die Aorta gepumpt, bis plötzlich die Zufuhr aufhört, sobald der Ventrikel entleert ist; wie in dem CautschucBallon muss auch hier ein negativer Druck entstehen und miissen die so eben angegebenen Folgen auftreten.

Beim Herzschlag hat man wirklich einen Zeitpunkt gefunden, in dem der Druck im Ventrikel negativ ist. Marey hat zuerst darauf aufmerksam gemacht und demselben den Namen ,vide postsystolique" gegeben.

Goltz und Gaule (Pflüger's Arch., Bd. XVII) haben ihn im lebenden Herzen selbst gemessen: sie fanden z. B. dass der Druck im linken Ventrikel bei einem kräftigen Hunde in der Herzperiode bis $-52 \mathrm{~mm}$ Quecksilber sinkt; sie fanden bei einem andern Hunde in der rechten Kammer - 17,2 mm. Die von ihnen gegebenen Curven beweisen zugleich, dass dieser negative Druck in den Ventrikeln nur kurze Zeit bei jedem Herzschlag dauert: dies folgt ja aus dem langsamen Sinken ihres Manometers, also erreicht das Quecksilberniveau erst nach einer grossen Zahl Herzschlägen seinen niedrigsten Stand.

Goltz und Gaule erklären den von ihnen in den Ventrikeln gemessenen negativen Druck ganz anders; sie sind der Meinung, er werde durch eine dem Herzen eigne Saugkraft verursacht, und er trete unmittelbar nach dem Aufhören der Systole also während der Diastole auf. - Nachher werde ich beweisen, dass der von ihnen gemessene negative Druck nicht während der Diastole des Ventrikels Statt finden kann. Weil jedoch unmittelbar nach dem Entleeren der Herzkammer und also indem sich die Ventrikelwand noch im contrahirten Zustande befindet (also während der Systole) ein kurzer negativer Druck darin bestehen muss, so sind die von Goltz und Gaule gefundenen Zahlen für das Mass des durch Blutbewegung entstandenen negativen Druckes zu halten.

Auch Mar ey erklärt den negativen Druck im Herzen für die Folge der Flüssigkeitsbewegung; da er jedoch ausser Acht gelassen hat, dass der Zeitpunkt, in dem der Ventrikel geleert ist, nicht mit dem Ende der Systole ïbereinstimmt, so entspricht allerdings der von ihm fälschlich gegebene Name "vide postsystolique“ Goltz und Gaule's Auffassung, im Wesen der Sache ist er aber ganz andrer Meinung als diese Forscher. 
2. Der Einfluss des leeren Raumes in der Herzkammer auf die Blutbewegung.

Es ist noch nicht entschieden, ob das Herz sich bei dessen Ausfüllung entweder ganz und gar passiv verhält oder dabei activ ist und durch seine Erschlaffung selbst die Kammerhöhle zu vergrössern sucht ${ }^{1}$ ). Es ist sehr wahrscheinlich, dass während der Diastole der Kammer eine Ausdehnung ihrer Höhle in Folge des Blutdruckes im coronären Gefässsystem Statt finden kann; die hierdurch erzeugte Kraft kann jedoch nur äusserst gering sein; aber es ist unerwiesen ob durch die Erschlaffung ihrer Wand selbst eine Saugkraft des Herzens verursacht werden kann.

Donders (Physiologie des Menschen, S. 46) sagt: „Der Ausdehnung des Herzens, bei welcher das Herz selbst sich passiv verhält, ......."; etwas weiter nimmt er freilich eine Saugkraft an: „Dazu“ (nämlich bei den Kräften die das Herz füllen) „kommt, für die Kammern wenigstens, die Elasticität der Herzsubstanz, welche sich alsbald geltend machen muss, so wie die Muskelfasern des Herzens erschlaffen".

Goltz und Gaule fanden, wie ich schon erwähnte, bei ihren Experimenten mit mehreren Thieren einen bedentenden negativen Druck im Herzen; so z. B. im linken Ventrikel des Hundes - $52 \mathrm{~mm}$ Quecksilber. Diesen negativen Druck leiten sie von der Elasticität der Ventrikelwand $a b$, die nachdem sie stark zusammengezogen war, plötzlich wie eine Feder auseinander schnellt, sobald die Contraction der Muskelfasern, die Systole also, geendet ist. Die Saugkraft des Ventrikels sollte sich also, ihrer Meinung nach, während der Diastole bemerklich machen, und die Kammerhöhle mit Blut aus dem Atrium füllen.

Es ist nicht unmöglich, dass durch Erschlaffung der Muskelwand einige Kraft ausgeübt werden kann; denn es ist sogar wahrscheinlich, dass die Muskelfasern, wenn sie aus dem contrahirten in den erschlafften Zustand übergehen, dabei einige Kraft èntwickeln zur Wiedererlangung ihrer primitiven Länge; diese Kraft ist jedoch so gering, dass man sie noch nicht hat messen können.

1) Der Einfluss der Respirationsphasen auf das Füllen des Herzens bleibt hier unerwähnt. Es gehört nicht hierher. 
Der erste Wellengipfel in dem absteigenden Schenkel der Pulseurve. 529

So wäre es ja auch nicht unmöglich, dass die Muskelfasern des Herzens die Ventrikel durch ihre Erschlaffung in geringem Grade vergrösserten; es ist mir jedoch unbegreiflich, wie Goltz und Gaule zur Erklärung des von ihnen gefundenen bedeutenden negativen Druckes, der erschlaffenden Muskelwand eine so grosse Kraft zuerkennen konnten.

Die Versuche, welche Ludwig Fick (Müller's Arch., 1849) mit dem ausgeschnittenen lebenden Katzenherzen angestellt hat, beweisen am deutlichsten, wie dabei von einer Saugkraft als Folge der Erschlaffung der Herzwand nicht die Rede sein kann; man soll aber diese sorgfältig angestellten und immer mit demselben Erfolg öfters wiederholten Experimente nicht kurzweg mis slungen heissen, wie Goltz und Gaule zu thun wagen.

Ich habe die statische Saugkraft des todten Herzens zu bestimmen versucht. Es wurden von einem Kalbsherzen, das in diastolischem Zustande erstarrt war, die Atria zugebunden, darauf wurde das eine Ende eines Glasröhrehens durch das Ostium aorticum hindurch in die Kammerhöhle geführt und die Aorta fest auf die Röhre gebunden; deren andres Ende senkrecht in ein mit Fliissigkeit gefiulltes Gefäss getaucht. Als nun der Ventrikel zusammengedrückt wurde und die Luft durch das Röhrchen entwich, stieg; sobald der Druck nachliess, die Flüssigkeit aus dem Gefäss in der Röhre auf: folglich ein Beweis, dass der linke Ventrikel dieses Herzens nach der Zusammenpressung eine eigne (statische) Saugkraft hatte, deren Werth einige Millimeter Quecksilber betrug.

Aus diesem Experimente soll man aber nicht folgern, als besässe das lebende Herz dieselbe Eigenschaft; die Elasticität der erstarrten Muskelwand muss ja eine ganz andere als die der lebenden Ventrikelwandung sein; will man die beim ausgeschnittenen Herzen erlangten Resultate auf das lebende Herz anwenden, so muss man mit einem unmittelbar nach dem Tode ausgeschnittenen und noch nicht erstarrten Herzen, dessen Muskelwandungen noch nicht verändert sind, den Versuch machen. Bei einem mit solchem Herzen angestellten Versuch findet man die Saugkraft der Ventrikelwand $=0$.

Noch ein andres Experiment habe ich mit dem Hunde angestellt, zum Beweis, dass der von Goltz und Gaule im rechten 
Ventrikel gefundene kurze aber bedeutende negative Druck (sie fanden dafür bei einem Hunde 17,2 mm Quecksilber) nicht während der Diastole der Herzkammer Statt gefunden haben kann. Wäre dies der Fall, wie unbegreiflich es auch sein möchte, und entstände also der negative Druck bei der Diastole des Ventrikels, so müsste man ja das Aufsaugen des Blutes aus dem Atrium in die Kammerhöhle wahrnehmen können, weil während der Diastole die Valvulae mitrales und tricuspidales entweder geöffnet sind oder sich wenigstens Nichts dem Erschliessen entgegenstellt.

Goltz und Gaule halten also den von ihnen gefundenen negativen Druck für eine Folge der grossen saugenden Kraft der Ventrikelwand. Die hierdurch entstandene negative Welle wird sich durch das Atrium hindureh bis in die grossen venösen Stämme verbreiten müssen und hier wahrgenommen werden. Zur Messung des dabei in der Vena cava entstandenen negativen Druckes, wurde bei einem Hunde eine mit einem Minimalmanometer verbundene Canuile in die Vena jugularis gefuihrt und deren offnes Ende bis in die Vena cava superior hinein geschoben. Der Thorax war geöffnet und die Respiration wurde kunstlich unterhalten. Trotz der starken Herzschläge sank das Niveau des Manometers nicht ${ }^{1}$ ): ein Beweis also dass kein Aufsaugen der Flüssigkeit durch den rechten Ventrikel Statt fand, obgleich man nach Goltz und Gaule einen negativen Druck von - 17,2 mm Quecksilber in diesem Ventrikel voraussetzen könnte. - Die Gleichheit zwischen rechtem und linkem Ventrikel berechtigt uns zur Anwendung dieses Ergebnisses auch auf letztern.

Aus dem hier angefuhthren Experimente ergibt sich unmittelbar, dass der von Goltz und Gaule in den Ventrikeln vorgefundene negative Druck nicht während der Diastole der Herzkammer Statt gefunden haben kann: folglich muss er während der Kammersystole auftreten und lässt sich nur aus der Fortbewegung des Blutes aus den Kammern in die grossen Schlagadern erklären.

Welchen Einfluss wird dann aber der negative Druck im Ventrikel auf die Blutbewegung ausïben?

1) Dieser Versuch wurde mit gleichem Erfolg mit zwei Hunden angestellt, 
Der erste Wellengipfel in dem absteigenden Schenkel der Pulscurve. 531

Es steht ausser Zweifel, dass das Abschliessen der Valvulae venosae während der Kammersystole nicht durch den Druck des Blutes auf die Kammerwand, sondern durch die zusammengezogenen Musculi papillares verursacht wird. Wäre ersteres der Fall, so miisste beim Beginne der Kammersystole ein bedeutender Theil des Inhaltes durch die weit geöffneten venösen Klappen, ehe diese sich schliessen, nach dem Atrium zuritickfliessen, was nicht wahrscheinlich ist. Dadurch würde auch der in der lebenden Vorkammer gehaltene Finger den Rüickschlag der Valvulae venosae fühlen, was nicht der Fall ist. Wahrscheinlich ist es jedoch, dass in Folge des hohen Blutdruckes in der Herzkammer jede Platte der Valvulae einigermasssen nach dem Atrium bombirt wird. Sobald jedoch der Ventrikel durch das Ausströmen des Blutes in die Aorta fast geleert ist und ein negativer Druck den positiven in Folge des wegströmenden Inhaltes ersetzt, werden die noch in der Kammerhöhle vorhandenen Bluttheile dadurch ebenfalls in die Aorta getrieben: der Ventrikel wird also durch die Systole vollkommen geleert.

Die innere Fläche der geschlossenen Valvulae venosae liegt nun gegen die Wand des contrahirten Ventrikels und wird in Folge des negativen Druckes sogar stark angedrückt. Weil die Musculi papillares stets zusammengezogen sind, bleiben die venösen Klappen trotz des negativen Druckes geschlossen; dieser negative Druck wird sogar den Verschlus s zwischen dem Atrium und der Ventrikelhöhle, der eigenthümlichen Klappeneinrichtung zufolge, befördern ${ }^{1}$ ).

Anstatt zur Füllung der Kammerhöhle beizutragen, wie dies in Marey's künstlichem Herzen Statt findet und wie Goltz und Gaule es beim lebenden Herzen voraussetzen, macht der negative Druek das Fïllen der Kammerhöhle aus dem Atrium heraus unmöglich.

Der negative Druck im Ventrikel hört sehr bald auf, hauptsächlich wegen des starken Aufeinanderpressens der Kanmerwandungen. Da jedoch während der Dauer jenes Druckes die Val-

1) Ganz anders ist dies in Marey's künstlichem Herzen, in welchem die Valvulae venosae durch eine einfache Klappe ersetzt sind, die in die künstliche Ventrikelhöhle getaucht ist und die sich bei jedem negativen Druck in dieser Höhle öffnet. 
vulae semilunares geöffnet sind, wird ein wenig Blut ans der Aorta nach dem Ventrikel zurïckgesogen; es kann jedoch nur sehr wenig in den Ventrikel hinein gerathen, weil die Valvulae semilunares sich gleich, gerade in Folge dieser Bewegung; schliessen mïssen: dieser Rückfluss erzeugt am Beginne der Aorta eine kleine positive Welle, die sich von hier aus die Schlagadern entlang peripheriseh fortpflanzt, und dies ist der Gipfel $s$ unserer Carotiscurve.

3. Anwendung des Vorhergehenden aufdie Carotiscurve.

Wir wollen schliesslich sehen, in wie fern die hier entwickelten Ideen durch die Pulscurve bestätigt werden.

Die Wellengipfel pflanzen sich von dem Beginne der Aorta mit einer gewissen Gesehwindigkeit in den Arterien fort; diese Fortpflanzungsgeschwindigkeit der Curvengipfel ist für jeden nicht vollk o mmen dieselbe (vgl. meine Pulscurve, S. 145), allein die Differenz ist so gering, dass die Wellenlinien der grossen dem Herzen nahen Schlagadern ohne Weiteres für das wahre Bild des am Ursprung der Aorta Stattfindenden zu halten sind.

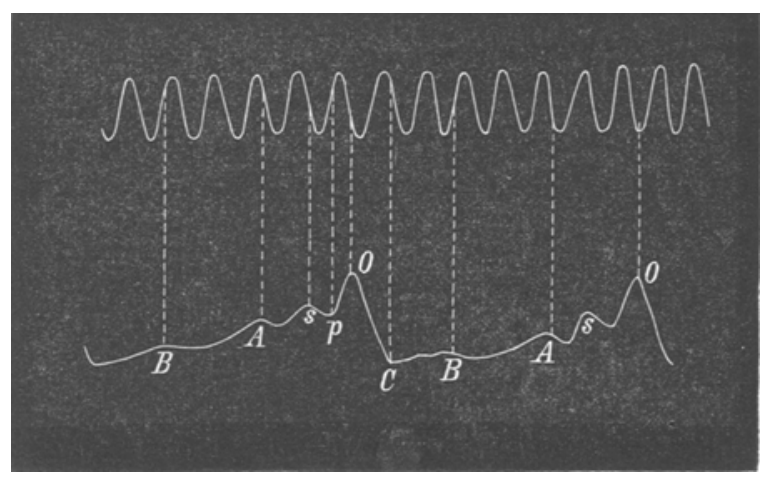

Fig' 3.

Wir mïssen also in jenem Theil der Curve, welcher der letzten Periode der Systole entspricht (in der also der Ventrikel entleert, aber noch contrahirt ist) finden: 1. ein Thal, 2. einen kleinen Gipfel, der diesem Thale unmittelbar folgt.

In dem hier gegebenen Tracé ist die Zeit vermittelst einer Stimmgabel, die zehn vollkommene Schwingungen auf 1 Sekunde macht, registrirt. 
Der erste Wellengipfel in dem absteigenden Schenkel der Pulscurve. 533

Der Punkt $C$ entspricht dem Beginne des in die Aorta gelangenden Blutes; da diese Periode cirea 0,1 Sek. dauert, so muss ihr Ende, d. h. der Anfang der letzten Periode der Systole zwischen $p$ und $o$ liegen. Diese letzte Periode, in welcher der linke Ventrikel geleert aber contrahirt ist, danert 0,115 Sek., wesshalb ihr Ende sich zwischen $s$ und $A$ befindet. Das Thal $p$ und der unmittelbar darauffolgende Gipfel $s$ liegen also in dem Theil der Wellenlinie, welcher der letzten Periode der linken Ventrikelsystole entspricht.

Die hier entwickelten Ideen werden also von der Wellenlinie der Carotis bestätigt und die Gipfel $p$ und $s$ zugleich erklärt:

$p$ ist also der in den Arterien fortgepflanzte negative Druck, der in der linken Ventrikelhöhle (in Folge der plotalichen Fortbewegung des in ihr enthaltenen Blutes) entsteht;

$s$ ist der fortgepflanzte Wellengipfel der an den Aortaklappen entsteht, wenn der negative Druck etwas Blut nach dem Ventrikel zurïcksaugt und dadurch jene Klappen sieh sehliessen.

Wie oben bei sehwa cher Contraction des Cautschukballons sich ein geringer negativer Druck (welcher der Fortbewegung der Flüssigkeit folgt) entwickelte, so musste dieser bei schwachen Herzcontractionen, z. B. bei abortiven Herzschlägen, ebenfalls gering sein; es kann jedoch durch dieses Saugen nur sehr wenig Blut nach dem Herzen zurïckgezogen werden, und deshalb kann die also entstandene positive Welle $s$ nur sehr klein sein. 